\title{
Estudio del potencial de bioconjugados Quantum Dots para el marcaje específico de dianas involucradas en Esclerosis Lateral Amiotrófica.
}

\author{
Nerea Baños Sánchez ${ }^{1,2, a}$, Carlota Tosat- Bitrián², Valle Palomo² \\ 1. Unidad de Bioquímica y Biología Molecular, Departamento de Biología de Sistemas, Facultad de Medicina y Ciencias \\ de la Salud, Universidad de Alcalá, 28871 Alcalá de Henares, Madrid, España. 2. Grupo de Biosensores y Química \\ Biológica, Centro de Investigaciones Biológicas- CSIC, Madrid, España. \\ a. nere11a@hotmail.com
}

Palabras clave: Multicolor Multicycle Molecular profiling (M3P); esclerosis lateral amiotrófica (ELA); Quantum Dots (QDs); inmunoensayo

\section{Resumen}

La investigación biomédica se encuentra en continuo avance y requiere la implantación de técnicas novedosas y prometedoras que permitan esclarecer mecanismos de enfermedades y seleccionar medicamentos eficaces para las mismas. El fin de estas técnicas es poder aplicar un tratamiento individualizado a cada paciente, es decir, implantar la medicina personalizada y para ello son necesarios biomarcadores y sensores efectivos. La técnica Multicolor Multicycle Molecular profiling (M3P), la cual se quiere aplicar en patologías de gran complejidad molecular como la esclerosis lateral amiotrofia (ELA), entre otras, utiliza Quantum Dots (QDs), nanopartículas con características fotoluminiscentes únicas y ventajosas sobre los fluoróforos tradicionales y con gran potencial como herramienta puntera en el campo de la biomedicina. En este trabajo se muestran los resultados obtenidos tras la realización de distintos inmunoensayos de QDs para estudiar su efectividad de marcaje de proteínas, la penetrabilidad en el núcleo y en consecuencia su potencial para seleccionar fármacos eficaces para patologías complejas como la ELA.

Cita: Baños Sánchez, Nerea; Tosat- Bitrián, Carlota; Palomo, Valle (2019) Estudio del potencial de bioconjugados Quantum Dots para el marcaje específico de dianas involucradas en Esclerosis Lateral Amiotrófica. dianas 8 (2): e201909fa08. ISSN 1886-8746 (electronic) journal.dianas.e201909fa08 http://www3.uah.es/dianas?e201909fa08. URI http://hdl.handle.net/10017/15181

Copyright: $\odot$ Baños-Sánchez N, Tosat--Bitrián C, Palomo V. Algunos derechos reservados. Este es un artículo open-access distribuido bajo los términos de una licencia de Creative Commons Reconocimiento-NoComercialSinObraDerivada 4.0 Internacional. http://creativecommons.org/licenses/by-nc-nd/4.0/

\section{Introducción}

Actualmente, en el campo de la biomedicina se están desarrollando técnicas para para el diagnóstico personalizado, las cuales serán de gran utilidad para el estudio de las redes moleculares que comprenden complejos procesos fisiológicos y patológicos. La técnica M3P promete convertirse en una herramienta clave en la investigación biomédica y diagnóstico clínico, así como asegura que poseerá un papel fundamental en la terapia dirigida para conseguir aplicar medicina personalizada dependiendo de las necesidades de cada paciente [1].

Es por ello que su empleo en patologías complejas resulta de gran utilizad, como en el caso de la ELA, una enfermedad neurodegenerativa que se caracteriza por la progresiva disminución y atrofia de masa neuromuscular debido a la pérdida de motoneuronas (MN) [2], del tronco espinal y del cortex motor [3]. Como ocurre en este tipo de enfermedades, los mecanismos moleculares que dan lugar a esta neurodegeneración no están claramente definidos en la actualidad. En 2006 se identificó la proteína TAR $D N A$ binding protein (TDP-43) como una de las moléculas responsables de esta enfermedad [4]. La proteína TDP-43 es codificada por el gen TARDBP, el cual pertenece a la familia de ribonucleoproteinas y presenta un dominio de unión al ARN/ADN muy conservado. TDP-43 posee dos dominios de unión al RNA; RRM1 y RRM2 y un dominio rico en glicina en el carboxilo terminal. Los dominios de unión al ARN son necesarios para la unión a los ácidos nucleicos mientras que el dominio carboxilo terminal favorece la unión de las proteínas. Es en este C- terminal donde se encuentran la mayoría de mutaciones identificadas en los casos de ELA familiar y esporádica, lo que se ha relacionado con el inicio temprano de la enfermedad y la rápida progresión de la misma. Además, numerosos estudios han descrito que se produce una unión anormal del ácido nucleico por un mutante denominado Q331K, lo que aumenta la tasa de agregación de TDP-43 y paralelamente se ha descubierto la importancia de esta proteína como componente crítico de la respuesta al daño del $\mathrm{ADN}$ en las neuronas motoras, por lo que desempeña un importante papel en la reparación del DNA, en concreto en la rotura de la doble hélice. De esta forma, en el ELA, la pérdida de función nuclear del TDP-43 desemboca en la reparación deficiente del ADN que se acumula, lo que produce un aumento de neurodegeneración. En condiciones fisiológicas, TDP-43 presenta una localización nuclear y se encuentra regulando muchas y diversas funciones implicadas en el 
procesamiento del ARN. En esta enfermedad disminuye su localización nuclear y se acumula en el citoplasma en forma de agregados [2]. En concreto aparece ubiquitinada e hiperfosforilada formando cuerpos de inclusión en el citoplasma de neuronas de la médula espinal de pacientes con ELA, pero también se ha detectado en pacientes con otras enfermedades neurodegenerativas como son la demencia frontotemporal y la enfemedad de Alzheimer. Dichas inclusiones están compuestas por formas poliubiquitinadas, hiperfosforiladas y fragmentos de TDP-43 [4].

Para poder ampliar el conocimiento de esta enfermedad y acercarse a la medicina personalizada, se requiere un gran avance en el diagnóstico clínico, donde es necesaria la existencia de sensores efectivos que ayuden a caracterizar la patología a nivel molecular en cada paciente de una forma individualizada. Las innovaciones en bio-nanotecnología están ampliando las capacidades de imágenes moleculares y en concreto las nanopartículas luminiscentes como los Quantum Dots (QDs), los cuales son una herramienta prometedora en el campo del análisis molecular debido a sus propiedades fotofísicas únicas, así como su biofuncionalización y las capacidades que presentan las sondas QDs- anticuerpo [5].

Los QDs son cristales de tamaño nanométrico formados por un núcleo metálico de selenio y cadmio $(\mathrm{CdSe})$ recubierto de sulfuro de Zinc $(\mathrm{ZnS})$. Estos materiales son en la actualidad una alternativa en su uso como sondas fluorescentes para la localización de diferentes estructuras biológicas [6] debido a que presentan un amplio espectro de absorción y estrecho de emisión, lo que se traduce en que pueden excitarse de forma simultánea distintos QDs empleando una fuente de luz común, detectándose así varias dianas moleculares que permiten establecer perfiles moleculares a nivel de célula única [5]. La longitud de onda a la que emite cada QD está relacionada con el tamaño del mismo, que oscila entre los 5-10 nm de diámetro [7]. Es por esto que presentan notables ventajas con respecto a los fluoróforos orgánicos, ya que son más estables, tienen un mayor coeficiente de extinción molar, así como un mayor tiempo de emisión de fluorescencia [8]. Asimismo, la superficie de los QDs comerciales están funcionalizadas con diversos grupos funcionales para poder llevar a cabo la conjugación de ligandos como es el ejemplo de ácidos nucleicos y proteínas, lo que permite su uso biológico en diferentes técnicas y con diversos fines. En el contexto de biomedicina e investigación biológica, los fluoróforos han sido utilizados tanto de forma general para tomar imágenes de células y tejidos o como conjugados con bioligandos específicos, en concreto cuando se conjugan con anticuerpos o ácidos nucleicos monocatenarios [8]. Además, Para hacer que estas nanopartículas sean solubles en agua y se puedan aplicar en técnicas biológicas, su superficie se modifica con ligandos hidrofílicos (como es el caso de polietilenglicol (PEG), o se encapsulan en polímeros anfifílicos $[6,9]$

a)

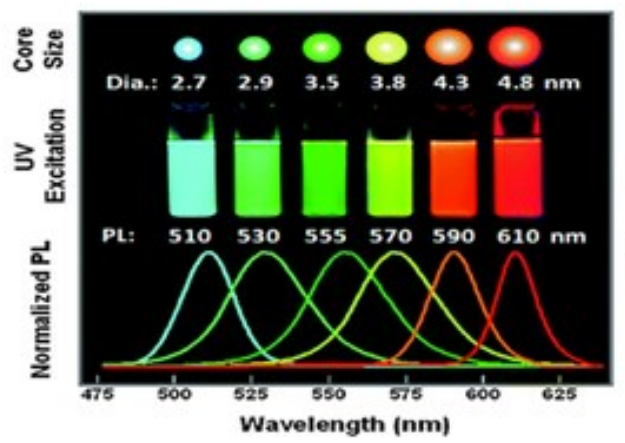

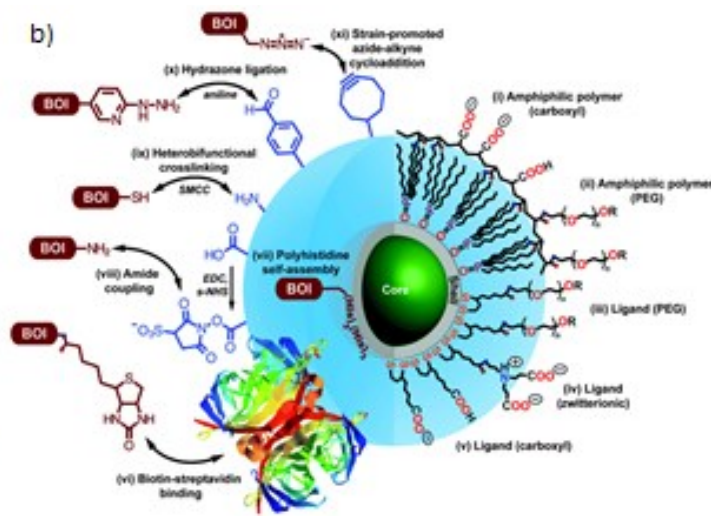

Figura 1.- a) Emisión de fluorescencia de los QDs en función de su tamaño b) Estructura de los QDs y diferentes estrategias de conjugación a biomoléculas [7].

Debido a estas características, los QDs han sido usados para el diagnóstico e investigación en proyectos tanto in vivo como in vitro de enfermedades, estructuras y procesos celulares [8]. Anticuerpos específicos que reconocen dianas biológicas concretas pueden ser conjugados con estos QDs para su uso en inmunofluorescencia, en la cual se permeabilizan las células con un detergente produciendo poros en la membrana de la célula, lo que permite a la sonda QD-proteína- anticuerpo el acceso y marcaje de la proteína de interés [6]. También se han diseñado protocolos para el desarrollo de sonda QD-Anticuerpo (QD-Ab) $[10,11]$ como una nueva alternativa de marcaje de estructuras biológicas en la que se puede prescindir de la proteína adaptora.

Algunos ejemplos de ensayos realizados con QD reafirman su importancia para el estudio molecular de patologías complejas y de diferentes naturalezas, por ejemplo el hallazgo del biomarcador CA-125 en pacientes con cáncer de ovario, el desarrollo de método de detección de antígenos de superficie específicos de Leishmania, el desarrollo de un sensor de detecta la interacción de clofazimina con las proteínas diana mediante el empleo de QD inmunoensayo, así como el reconocimiento de proteínas como heparina, transferrina, fosfatasa alcalina (ALP), entre otros. Estos ejemplos ponen de manifiesto la necesidad de seguir ampliando el conocimiento sobre los QDs como biomarcadores en una amplia variedad de enfermedades [12]. 
El objetivo de este trabajo es implementar un inmunoensayo con QD tanto en células SH-SY5Y como la línea celular HeLa con la finalidad de estudiar la potencialidad de estos sensores para marcar dianas clave de la enfermedad de ELA. Para ello se comparará la penetrabilidad de los QD en el núcleo mediante el marcaje de diferentes proteínas nucleares y citosólicas con la finalidad última de poder cuantificar los niveles de TDP-43 y pTDP-43 dentro y fuera del núcleo para poder establecer la patología molecular del ELA. Para ello se realizarán diferentes protocolos de bioconjugación de QD con proteína G y SpA con el anticuerpo y QD-Ab unidos de forma covalente directa.

\section{Materiales y métodos}

\section{Equipos y servicios}

Para el desarrollo de los experimentos llevados a cabo en este trabajo, se utilizaron distintos equipos del Centro de Investigaciones Biológicas del CSIC. Las imágenes de fluorescencia se recogieron en un microscopio confocal láser (CLSM) LEICA TCS SP5 en el servicio de microscopía láser confocal, la concentración de los QDs, y los bioconjugados QDs-PG/QDs-SpA y QD-Ab se midió en un espectrofotómetro (Ultrospec 2100, Amersham Bioscience), y los ensayos cinéticos se llevaron a cabo en la máquina Blitz de la casa comercial FortéBio en el servicio de interacciones moleculares.

\section{Síntesis de bioconjugados de QD-PG y QD-SpA}

Para la síntesis de los bioconjugados, con proteínas adaptoras QD-PG y QD-SpA, se utilizaron QDs comerciales (QD $655 \mathrm{~nm}$, Invitrogen, $\mathrm{n}^{\circ}$ cat Q21521MP, QD $565 \mathrm{~nm}$ Invitrogen, $\mathrm{n}^{\circ}$ cat Q21531MP), con picos de emisión centrados a $565 \mathrm{~nm}$ y $655 \mathrm{~nm}$ ( QD565, QD655). Previo al empleo de los QDs, se centrifugó el stock de QDs $8 \mu \mathrm{M}$ a $2000 \mathrm{rpm} 1$ minuto a temperatura ambiente (TA) con el fin de eliminar los posibles agregados que se hubieran formado. Posteriormente, éstos se activaron con BS3 (3-sulfo- $N$ hidroxisuccinimida del ácido subérico), $\mathrm{n}^{\circ}$ cat S5799 Sigma-Aldrich). Se preparó en un eppendorf las siguientes cantidades: $25 \mu \mathrm{L}$ de $8 \mu \mathrm{M}$ QD stock, $61 \mu \mathrm{L}$ de agua, $10 \mu \mathrm{L}$ de PBS 10X, $4 \mu \mathrm{L}$ de una solución $50 \mathrm{mM}$ BS3. Se obtuvo así $100 \mu \mathrm{L}$ de QDs a $2 \mu \mathrm{M}$ en PBS con un exceso de 1000x de BS3. Se incubaron durante 30 minutos a TA. Una vez activados, los QDs se purificaron mediante el empleo de una columna NAP-5 ( $\mathrm{n}^{\mathrm{o}}$ cat 17085301, GE Healthcare) pre-equilibrada con PBS 1X (pH 7,5). El avance de los QDs se siguió mediante una lámpara portátil UV. Dependiendo del bioconjugado obtenido, se recogieron entre 400-600 $\mu \mathrm{L}$ de QDs, los cuales se concentraron hasta 30-40 $\mu \mathrm{L}$ utilizando filtros Amicon Ultra $100 \mathrm{kDa}$ MWCO ( ${ }^{\circ}$ cat UFC510024, Millipore) por centrifugación a TA, 7 min $7500 \mathrm{rpm}$. Posteriormente se conjugaron de forma covalente con $100 \mu \mathrm{L}$ de proteína SpA (Proteína A de Staphylococcus aureus, $\mathrm{n}^{\circ}$ cat P6031, Sigma-Aldrich) o Proteína G (Proteína G de Strepyococcus $n^{\circ}$ cat 08062-5MG-F, Sigma-Aldrich) respectivamente, ambas a una concentración $100 \mu \mathrm{M}$. La reacción se incubó durante toda la noche a TA y se llevaron a cabo 6 centrifugaciones de min a $75000 \mathrm{rpm}$, llevando el bioconjugado a un volumen final de $100 \mu \mathrm{L}$ con PBS $1 \mathrm{X}$.

Una vez sintetizados, se calculó su concentración final con respecto a una muestra a $1 \mu \mathrm{M}$ (preparada a partir del QD stock $8 \mu \mathrm{m}$ ), mediante la medida de la absorbancia en el espectrofotómetro. Los conjugados preparados se muestran en la Tabla 1.

\begin{tabular}{|c|c|c|c|c|}
\hline Código & $\Lambda$ emisión & Casa comercial & Concentración $\mu \mathrm{M}$ & Proteína adaptora \\
\hline NBS 1.07 & 565 & Invitrogen Q21531MP & 1 & PG \\
\hline NBS 1.14 & 655 & Invitrogen Q21521MP & 1 & PG \\
\hline NBS 1.17 & 565 & Invitrogen Q21531MP & 1 & PG \\
\hline NBS 1.20 & 565 & Invitrogen Q21531MP & 0,9 & PG \\
\hline NBS 1.25 & 565 & Invitrogen Q21531MP & 0,77 & SpA \\
\hline NBS 1.27 & 655 & Invitrogen Q21521MP & 0,95 & $\mathrm{SpA}$ \\
\hline NBS 1.29 & 655 & Invitrogen Q21521MP & 0,5 & PG \\
\hline NBS1.39 & 655 & Invitrogen Q21521MP & 0,8 & SpA \\
\hline
\end{tabular}

Tabla 1.- Descripción de los bioconjugados QD-PG y QD-SpA sintetizados.

Una vez conjugado el QD con la proteína adaptora, se incubó la sonda QD-Ab añadiendo en un eppendorf de 1,5 mL 2,5 PBS 1X, 1,5 $\mu \mathrm{L}$ de anticuerpo primario monoclonal a una concentración de $0,2 \mathrm{mg} / \mathrm{mL}$ y 6 $\mu \mathrm{L}$ del bioconjugado QD-SpA o QD-PG a una concentración de $1 \mu \mathrm{M}$ con el anticuerpo correspondiente durante una hora (Tabla 2). 


\begin{tabular}{|c|c|c|c|c|}
\hline Código & $\Lambda$ emisión & Casa comercial & Bioconjugado & $\begin{array}{c}\text { Anticuerpo } \\
\text { conjugado }\end{array}$ \\
\hline NBS $1.07 a$ & 565 & $\begin{array}{l}\text { Invitrogen } \\
\text { Q21531MP }\end{array}$ & NBS 1.07 & $1,5 \mu \mathrm{L}$ histona \\
\hline NBS $1.07 b$ & 565 & $\begin{array}{l}\text { Invitrogen } \\
\text { Q21531MP }\end{array}$ & NBS 1.07 & $1,5 \mu \mathrm{L}$ tubulina \\
\hline NBS $1.07 c$ & 565 & $\begin{array}{l}\text { Invitrogen } \\
\text { Q21531MP }\end{array}$ & NBS 1.07 & $0,5 \mu \mathrm{L}$ histona \\
\hline$N B S 1.14 a$ & 655 & $\begin{array}{l}\text { Invitrogen } \\
\text { Q21521MP }\end{array}$ & NBS 1.14 & $1,5 \mu \mathrm{L}$ histona \\
\hline NBS $1.14 b$ & 655 & $\begin{array}{l}\text { Invitrogen } \\
\text { Q21521MP }\end{array}$ & NBS 1.14 & $1,5 \mu \mathrm{L}$ tubulina \\
\hline$N B S 1.14 c$ & 655 & $\begin{array}{l}\text { Invitrogen } \\
\text { Q21521MP }\end{array}$ & NBS 1.14 & $0,5 \mu \mathrm{L}$ histona \\
\hline$N B S 1.14 d$ & 655 & $\begin{array}{l}\text { Invitrogen } \\
\text { Q21521MP }\end{array}$ & NBS 1.14 & $1,5 \mu \mathrm{L}$ histona \\
\hline NBS $1.14 e$ & 655 & $\begin{array}{l}\text { Invitrogen } \\
\text { Q21521MP }\end{array}$ & NBS 1.14 & $1,5 \mu \mathrm{L}$ tubulina \\
\hline NBS $1.17 a$ & 565 & $\begin{array}{l}\text { Invitrogen } \\
\text { Q21531MP }\end{array}$ & NBS 1.17 & $1,5 \mu \mathrm{L}$ histona \\
\hline NBS $1.17 b$ & 565 & $\begin{array}{l}\text { Invitrogen } \\
\text { Q21531MP }\end{array}$ & NBS 1.17 & 1,5 $\mu \mathrm{L}$ Tubulina \\
\hline$N B S 1.25 a$ & 565 & $\begin{array}{l}\text { Invitrogen } \\
\text { Q21531MP }\end{array}$ & NBS 1.25 & $1,5 \mu \mathrm{L}$ histona \\
\hline NBS $1.29 a$ & 655 & $\begin{array}{l}\text { Invitrogen } \\
\text { Q21521MP }\end{array}$ & NBS 1.29 & $1,5 \mu \mathrm{L}$ histona \\
\hline$N B S 1.29 b$ & 655 & $\begin{array}{l}\text { Invitrogen } \\
\text { Q21521MP }\end{array}$ & NBS 1.29 & $1,5 \mu \mathrm{L}$ tubulina \\
\hline
\end{tabular}

Tabla 2.- Descripción de las sondas QD-PG-Ab y QD-PG-Ab sintetizadas.

\section{Síntesis de bioconjugados covalentes QD-Ab}

Para la síntesis de bioconjugados de QD-Ab de forma covalente directa, se unen los anticuerpos a la superficie de los QDs sin necesidad de la proteína adaptora. Para ello se pueden hacer reaccionar los grupos amino de los QD a los grupos carboxilo del anticuerpo, o se puede utilizar un conector como BS3 para conjugar los grupos amino de los QDs y unirlos a los grupos amino del anticuerpo. En un primer lugar se activaron los grupos carboxilo del anticuerpo (cuyo ratio con respecto al QD fue 1:5, 1:10, 1:15, 1:20, 1:25) mediante el empleo de 10.000 equivalentes de EDC (1-Ethyl-3-[3-dimethylaminopropyl] carbodiimide hydrochloride) y NHS (N-hydroxysulfosuccinimide) en PBS $\mathrm{mM}(\mathrm{pH}=7,4)$ hasta un volumen final de $46 \mu \mathrm{L}$ durante 30 minutos con la finalidad de unir este grupo funcional con el grupo amina del QD y formar así un enlace amida. Posteriormente se añadieron $4 \mu \mathrm{L}$ del Stock $8 \mu \mathrm{M}$ de QD y se incubó durante 4 horas en agitación a TA. Pasadas 4 horas se llevó a cabo la purificación del bioconjugado mediante la columna NAP-5, se centrifugó para concentrar el bioconjugado y se midió la concentración de los mismos (Tabla 3).

\begin{tabular}{|c|c|c|c|c|}
\hline Código & $\Lambda$ emisión & Casa comercial & Concentración $\mu \mathrm{M}$ & Anticuerpo \\
\hline NBS1.31 & 655 & Invitrogen Q21521MP & 0,34 & $\begin{array}{c}\text { Histona (1:5 respecto al } \\
\text { QD) }\end{array}$ \\
\hline$N B S 1.31 \mathrm{~A}$ & 655 & Invitrogen Q21521MP & 0,33 & $\begin{array}{c}\text { Histona( 1:10 respecto al } \\
\text { QD) }\end{array}$ \\
\hline$N B S 1.31 B$ & 655 & Invitrogen Q21521MP & 0,24 & $\begin{array}{c}\text { Histona( (1:15 respecto al } \\
\text { QD) }\end{array}$ \\
\hline NBS1.31C & 655 & Invitrogen Q21521MP & 0,34 & $\begin{array}{c}\text { Histona( 1:20 respecto al } \\
\text { QD) }\end{array}$ \\
\hline NBS1.31D & 655 & Invitrogen Q21521MP & 0,25 & $\begin{array}{c}\text { Histona( 1:25 respecto al } \\
\text { QD) }\end{array}$ \\
\hline$N B S 1.32$ & 655 & Invitrogen Q21521MP & 0,3 & $\begin{array}{c}\text { Tubulina( } 1: 5 \text { respecto al } \\
\text { QD) }\end{array}$ \\
\hline
\end{tabular}

Tabla 3.- Descripción de los bioconjugados QD-Ab sintetizados.

En la segunda estrategia planteada, utilizando el conector BS3, se activó el QD ( $25 \mu \mathrm{L}$ del stock $8 \mu \mathrm{M})$ con $4 \mu \mathrm{L}$ de BS3 el QD que está funcionalizado en amina. Posteriormente se hicieron reaccionar el QD y EDC hasta un volumen de $11 \mu \mathrm{L}$, teniendo en cuenta que el ratio EDC / QD era $600 \mathrm{nmol} / 120 \mathrm{pmol}$. La mezcla se incubó durante 5 minutos a TA y posteriormente se añadió el anticuerpo (teniendo en cuenta el 
ratio QD-BS3/ anticuerpo es 120pmol/6pmol), $10 \mu \mathrm{L}$ PBS 10x, y agua hasta un volumen de $50 \mu \mathrm{L}$. La reacción se incubó durante 1 hora y media en condiciones de TA en una rueda giratoria y oscuridad [8, 11]. Los conjugados obtenidos y su anticuerpo correspondiente se refleja en la Tabla 4.

\begin{tabular}{|c|c|c|c|c|}
\hline Código & $\Lambda$ emisión & Casa comercial & Concentración $\boldsymbol{\mu} \mathrm{M}$ & Anticuerpo \\
\hline NBS1.34 & 655 & Invitrogen Q21521MP & 0,73 & Histona \\
\hline$N B S 1.34 A$ & 655 & Invitrogen Q21521MP & 0,67 & Histona \\
\hline$N B S 1.34 B$ & 655 & Invitrogen Q21521MP & 0,56 & Histona \\
\hline NBS1.36 & 655 & Invitrogen Q21521MP & 0,63 & GAPDH \\
\hline NBS1.37 & 655 & Invitrogen Q21521MP & 0,66 & $\begin{array}{c}\text { Anticuerpo secundario } \\
\text { Anti-mouse }\end{array}$ \\
\hline
\end{tabular}

Tabla 4.- Descripción de los bioconjugados QD- BS3- Ab sintetizados.

Caracterización de los bioconjugados QD- PG, QD- SpA y QD- Ab

\section{Movilidad electroforética.}

Los bioconjugados QD-PG, QD-SpA y QD-Ab se caracterizaron en gel de agarosa al 1\%, aplicando una corriente de $110 \mathrm{~V}$ durante 20- $25 \mathrm{~min}$. Dependiendo de la concentración de los bioconjugados, se emplearon entre 2,5- $5 \mu \mathrm{L}$ de los mismos, $5 \mu \mathrm{L}$ de QD $1 \mu \mathrm{m}$ y se añadió a ambos $1 \mu \mathrm{L}$ de tampón de carga $\left(\mathrm{n}^{\mathrm{o}}\right.$ cat G2526, Sigma-Aldrich) y se cargaron en el gel. Las imágenes se tomaron en el equipo de quimioluminiscencia (Chemidoc Imaging System, Bio-rad).

\section{Cultivo celular y procesamiento}

Para los ensayos llevados a cabo, se utilizaron líneas celulares de neuroblastoma humano SH-SY5Y así como de la línea celular inmortalizada HeLa. La línea celular SH-SY5Y fue previamente fijada en paraformaldehido (PFA) al 4\%. En el caso de la línea celular HeLa, el PFA utilizado fue al 2\% dado que se comprobó en la literatura que era el porcentaje óptimo de fijación de estas líneas celulares [13].

\section{Ensayo cinético}

Se llevaron a cabo ensayos cinéticos para comprobar la unión entre la PG con el anticuerpo, el protocolo a seguir fueron las indicaciones del manual FortéBIO.

Para el desarrollo del ensayo cinético, se emplearon biosensores de amina reactiva de segunda generación (AR2G), los cuales tienen una alta densidad de grupos carboxílicos que fueron activados con EDC stock en agua a una concentración $400 \mathrm{mM}$ y s-NHS stock en agua a una concentración $200 \mathrm{nM}$. Previamente se estudió el $\mathrm{pH}$ al que la unión era más eficiente $(\mathrm{pH}=5)$. Una vez activado el biosensor, se inmovilizó la proteína $\mathrm{G}(100 \mu \mathrm{g} / \mathrm{mL}$ en tampón acetato $10 \mathrm{mM}$ a $\mathrm{pH}=5)$. Se estudió su unión con el anticuerpo de tubulina y de histona a diferentes concentraciones $70,120,150,200 \mathrm{nM}$ para poder establecer la constante de disociación $(\mathrm{Kd})$ con el fin de hallar la afinidad con la que se está uniendo el anticuerpo a la proteína $\mathrm{G}$.

\section{Inmunofluorescencia}

Previo a la realización del experimento se procedió a la preparación de los Buffers necesarios para el desarrollo del mismo. Las cantidades que aquí figuran son las necesarias para tratar 10 pocillos de una placa de 24 pocillos.

Tampón de permeabilización. Se prepararon $5 \mathrm{~mL}$ de Tritón-X-100 0,25\% tras mezclar 4,4 mL de agua, $0,125 \mathrm{~mL}$ Triton-X-100 10\% y 0,5 mL PBS $10 \mathrm{X}$.

Tampón de bloqueo. Se emplearon13,2 mL agua, 1,5 mL PBS 1X, $300 \mathrm{mg}$ BSA (Bovine Serum Albumin, $\mathrm{n}^{\circ}$ cat 10735078001 , Sigma-Aldrich) y $300 \mu \mathrm{L}$ caseína $5 \%$ ( $\mathrm{n}^{\circ}$ cat $\mathrm{C} 4765$, Sigma-Aldrich) obteniendo una solución de BSA $2 \%$.

Tampón de marcaje. Se mezclaron 2,7 mL de agua, 0,3 mL de PBS 10X y $180 \mathrm{mg}$ de BSA obteniendo una solución de BSA al 6\%.

Tampón de lavado. Se prepararon $20 \mathrm{~mL}$ de tampón de lavado, tras mezclar 17,6 $\mathrm{mL}$ de agua, $2 \mathrm{~mL}$ de PBS 10X, $200 \mathrm{mg}$ BSA y $400 \mu \mathrm{L}$ de caseína 5\%.

Las células previamente fijadas se permeabilizaron con $500 \mu \mathrm{L}$ de tampón de permeabilización durante 20 min con DTAC, se lavaron 3 veces con PBS 1x,5 minutos cada lavado y posteriormente se añadió el 
TX100 durante 5 min a TA. En el caso de las células permeabilizadas con TX100, este se añadió durante 25 min a TA. Una vez permeabilizadas, se lavaron las células 3 veces con PBS 1X y se bloquearon con $500 \mu \mathrm{L}$ de tampón de bloqueo durante $30 \mathrm{~min}$ a TA. Se aspiró el tampón de bloqueo y se añadió la sonda QD-Ab diluida en $300 \mu \mathrm{L}$ de tampón de marcaje, consiguiendo una dilución 10:300. Las células se incubaron durante dos horas y media a TA. Pasado este tiempo, se retiró la sonda QD-Ab y se añadieron $200 \mu \mathrm{L}$ de HCS nuclear mask DeepRed ( $n^{\circ}$ cat H10294, ThermoFisher) durante 30 minutos.

A continuación, se aspiró la nuclear mask y se lavaron las células con $1 \mathrm{~mL}$ de tampón de lavado dos veces consecutivas, dejando la segunda $10 \mathrm{~min}$. Tras 3 lavados de 5 min cada uno con $1 \mathrm{~mL}$ de PBS $1 \mathrm{X}$ por pocillo, los cristales se montaron en los portaobjetos con medio Fluoromount $\left(\mathrm{n}^{\circ}\right.$ cat F4680, SigmaAldrich) y se guardaron a TA durante 16 horas.

Como control positivo, se realizó una inmunofluorescencia utilizando un anticuerpo secundario marcado con Alexa Fluor 488 (0,75 mg/mL, $\mathrm{n}^{\circ}$ cat 115-546-062, Jackson Inmuno Research). Se siguió el mismo protocolo de permeabilización y bloqueo que en el caso del inmunoensayo con QDs. El anticuerpo primario se utilizó a una dilución 1:300 en tampón de marcaje y se incubó durante $1 \mathrm{~h}$ a TA. Las células se lavaron 3 veces con PBS 1X y posteriormente se añadió el anticuerpo secundario diluido 1:600 en 300 $\mu \mathrm{L}$ de tampón de marcaje y se incubó $1 \mathrm{~h}$ a TA. Como controles negativos, se siguió el mismo protocolo incubando las células con $6 \mu \mathrm{L}$ a $1 \mu \mathrm{M}$ sin el empleo de anticuerpo primario.

Las imágenes se obtuvieron en el microscopio confocal Leica TCS SP5 con un objetivo de aumento 63. Los láseres de excitación utilizados fueron 405 y 488. Los rangos de fluorescencia de cada sonda QDSpA y QD-PG fueron establecidos previamente [14].

\begin{tabular}{c|c|c|}
\hline Anticuerpo & Casa comercial & Referencia \\
\hline Tubulina & Santa Cruz & Sc-5286 \\
\hline Histona & Santa Cruz & Sc-8030 \\
\hline GAPDH & ProteinTech & $10494-1-$ AP \\
\hline
\end{tabular}

Tabla 5.- Especificaciones de los anticuerpos utilizados en este estudio.

\section{Resultados}

Para analizar el potencial de las sondas QDs en el esclarecimiento de las bases moleculares de la ELA, se decidió estudiar bioconjugados unidos a anticuerpos primarios monoclonales y en concreto los QDs se unieron a histona y tubulina para determinar su marcaje intracelular.

Para generar los bioconjugados, se unió al QD una proteína adaptora (SpA o PG), que se unió de forma posterior al anticuerpo primario monoclonal. Tras la reacción y posterior purificación, se llevó a cabo la caracterización de dichos bioconjugados en gel de agarosa al $1 \%$.

\section{Caracterización de los bioconjugados QD-PG Y QD-SpA}

La bioconjugación de los QD con la PG y SpA se confirmó determinando la migración electroforética de los QD libres y bioconjugados en un gel de agarosa al 1\%. Los resultados obtenidos muestran que los QDs libres presentan una movilidad electroforética menor que los QDs conjugados. Esta mayor movilidad podría ser explicada por el aumento de cargas negativas en la superficie del QD por incorporación de las proteínas adaptoras SpA y PG que presentan aminoácidos negativos glutámico (Glu) y aspártico (Asp). En concreto, la proteína SpA tiene notablemente mayor número de Glu y Asp con respecto a la PG (Figura 1).

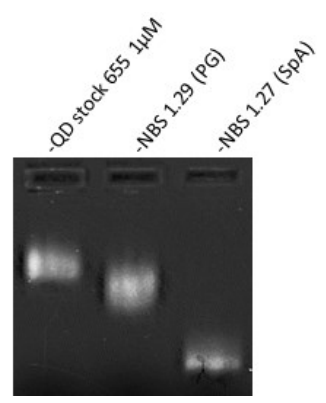

Figura 2.-Movilidad electroforética de los diferentes bioconjugados con respecto al QD stock en el gel de agarosa al $1 \%$. 
Los conjugados sintetizados por reacción covalente directa también se analizaron por movilidad electroforética y de nuevo se observó una mayor migración de los QDs conjugados con el anticuerpo con respecto al QD sin conjugar.

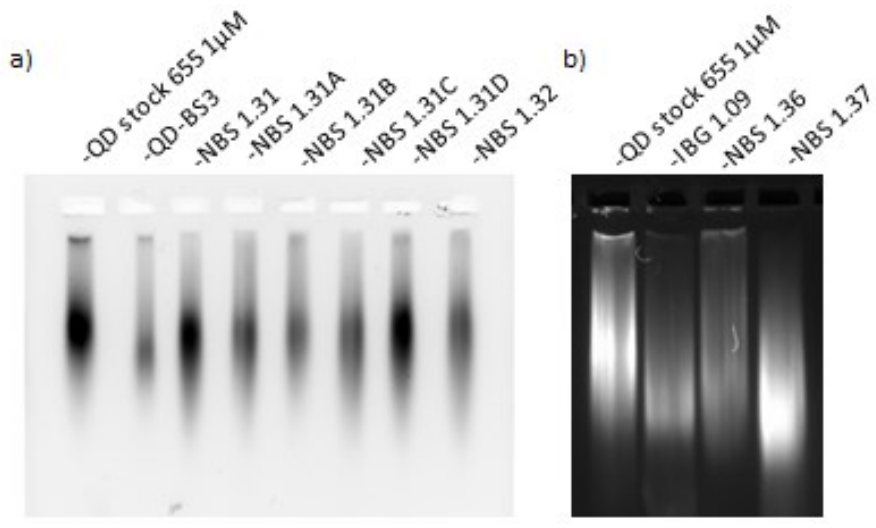

Figura 3.- a) Movilidad electroforética del QD stock $6551 \mu \mathrm{M}$, QD unido a BS3 y los bioconjugados sintetizados mediante el protocolo de activación del anticuerpo (histona a diferentes diluciones y Tubulina) con EDC y NHS y posterior incubación con el QD. b) Movilidad electroforética del QD stock $6551 \mu \mathrm{M}$ y los bioconjugados sintetizados mediante el protocolo de activación de QD-BS3 con EDC y NHS y posterior incubación con el anticuerpo.

\section{Ensayo cinético}

Una vez comprobada la bioconjugación del QD con la proteína y anticuerpos respectivamente, se comprobó si la proteína adaptora se unía correctamente al anticuerpo tubulina e histona. Para ello se llevó a cabo un ensayo cinético, obteniendo así la Kd tubulina= 1, 899e -9 M y Kd histona=2,625e-9 M y confirmando así su unión en ambos casos.

Debido a que el objetivo del trabajo es analizar la capacidad que tienen los QDs para marcar tanto dianas citosólicas como nucleares y así poder establecer el mecanismo de acción patológico de TDP-43, se estudió la capacidad de penetración de las sondas QDs en el citosol y núcleo. Para ello se decidió utilizar distintos detergentes en el protocolo de permeabilización celular previo a los ensayos de inmunofluorescencia.

\section{Inmunoensayo con QD en la línea celular SH-SY5Y}

En primer lugar, se estudió el posible efecto de los diferentes detergentes sobre la diana tubulina. Para ello se emplearon DTAC (surfactante iónico), TX100 (surfactante no iónico) y SDS (surfactante aniónico) con el fin de descartar la posible degradación de esta proteína citosólica con cada uno de dichos detergentes. Se comprobó por inmunofluorescencia la integridad de la tubulina.

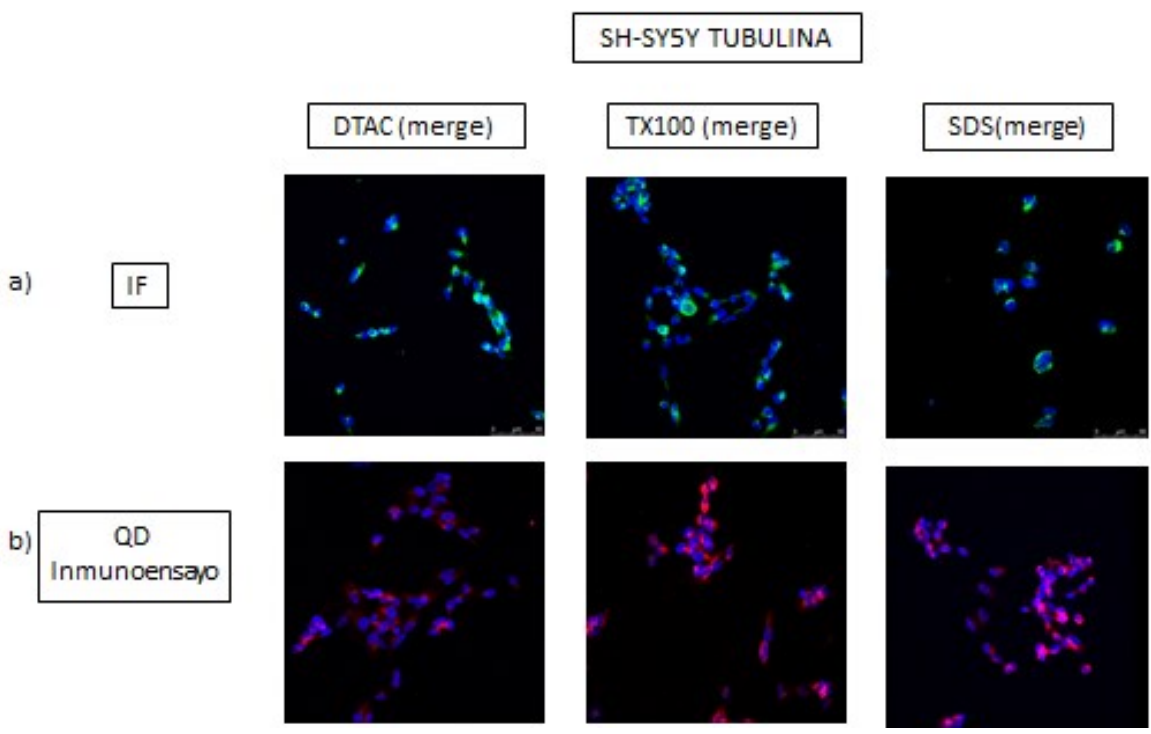

Figura 4.- Marcaje específico de tubulina utilizando como detergentes DTAC, TX100 y SDS en la línea celular SH-SY5Y. a) Inmunofluorescencia clásica en dos pasos. Marcaje específico de tubulina en células SH-SY5Y con anticuerpo secundario Alexa-488. b) Inmunoensayo basado en QD. Marcaje de tubulina con DTAC, TX100 y SDS en las células SH-SY5Y con la sonda CBT2.14. La escala es $50 \mu \mathrm{m}$. 
Con este ensayo también se pudo comprobar que los QDs marcaban eficazmente esta diana citosólica, obteniendo imágenes similares a las de IF clásica en dos pasos (Figura 4).

Posteriormente, se quiso estudiar la penetrabilidad de los QD en el núcleo de las células, llevando a cabo el marcaje de histona para comprobar que estos accedían a nivel nuclear. En las imágenes obtenidas no se observa marcaje nuclear tras la incubación con los QDs, lo que indica que las nanopartículas no están penetrando en núcleo (Figura 5).

a)
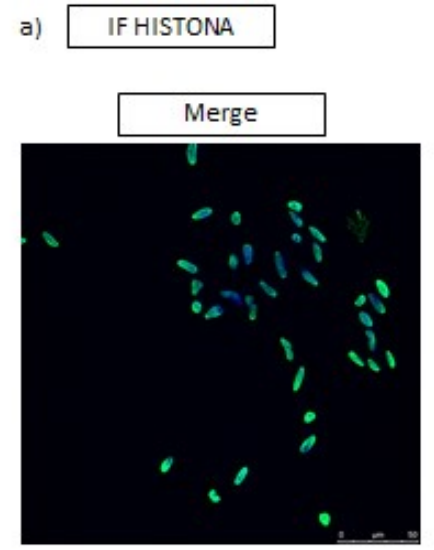

b) QD INMUNOENSAYO

Figura 5.- Marcaje específico de histona en la línea celular SH-SY5Y. a) inmunofluorescencia clásica en dos pasos. Marcaje específico de histona en células SH-SY5Y con anticuerpo secundario Alexa488.b) Inmunoensayo basado en QD. Marcaje de histona con TX100 como detergente en condiciones normales de incubación del QD con el anticuerpo (CTB2.19F) y con DTAC como detergente, durante 12 horas (NBS.125).

\section{Inmunoensayo con QD en la línea celular HeLa}

Debido a la ausencia de marcaje nuclear por parte de las sondas QD-proteína- anticuerpo, se plantearon diversas hipótesis que condujeron a la realización de nuevas estrategias de bioconjugación de QDs, esta vez mediante su unión directa al anticuerpo por activación en primer lugar de los grupos carboxílicos del anticuerpo que reaccionaron con las aminas de los QDs, y en segundo lugar mediante la funcionalización en amino de los QDs con BS3 y posterior activación de los mismos que reaccionarían con las aminas del anticuerpo.

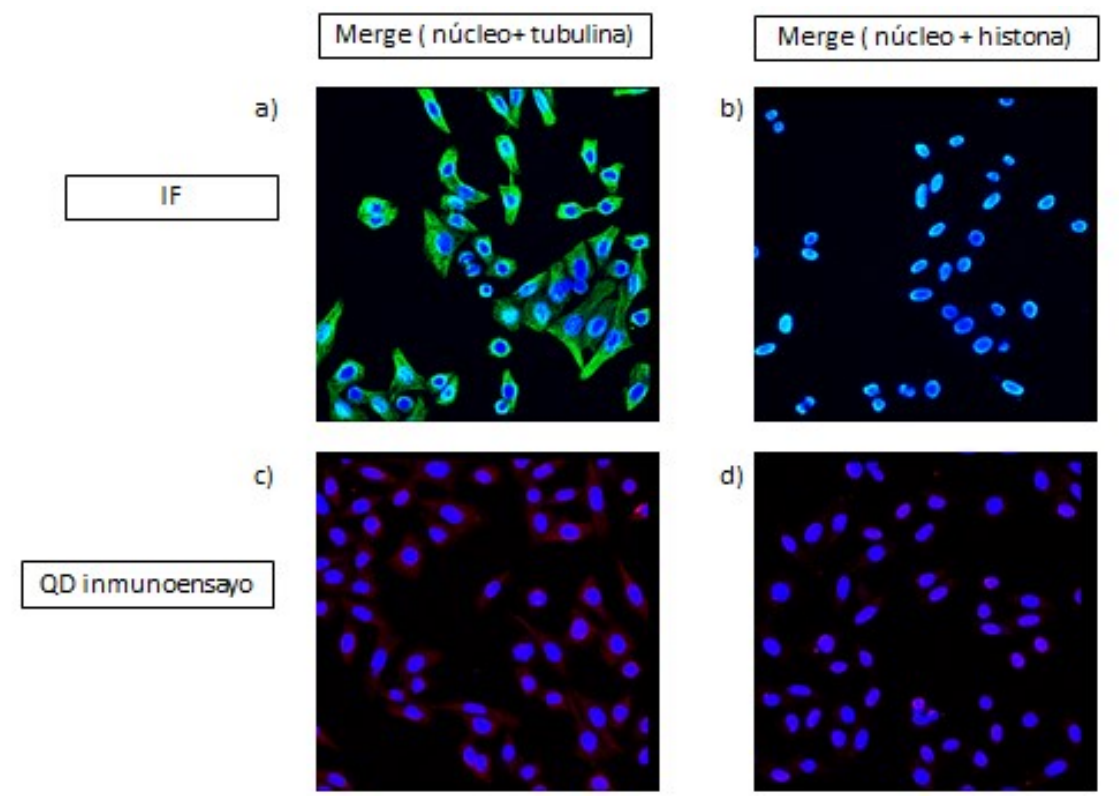

Figura 6.- Marcaje específico de tubulina e histona en la línea celular HeLa. a) Inmunofluorescencia clásica en dos pasos. Marcaje específico de Tubulina en células HeLa con anticuerpo secundario Alexa-488. b) Inmunofluorescencia clásica en dos pasos. Marcaje específico de histona en células HeLa con anticuerpo secundario Alexa-488.c) Inmunoensayo basado en QD. Marcaje de Tubulina empleando DTAC como detergente, sonda CTB 2.22. d) Inmunoensayo basado en QD. Marcaje de histona empleando DTAC como detergente, sonda CTB 2.12.

Por otro lado, se decidió utilizar otra línea celular, en este caso la línea HeLa. Se llevó a cabo una inmunofluorescencia clásica para comprobar que se marcaban correctamente tubulina e histona $\mathrm{y}$ paralelamente se llevó a cabo un inmunoensayo de QD marcando tubulina e histona. Con la nueva línea 
celular de nuevo se observó marcaje eficaz de tubulina pero no de histona, confirmando la eficacia de los QDs para marcar dianas citosólicas pero no nucleares (Figura 6).

Al no observar diferencias significativas del marcaje de histona por parte de las sondas QDs, se procedió al empleo de los nuevos bioconjugados $\mathrm{QD} / \mathrm{Ab}$, que tampoco demostraron una mayor eficacia en el marcaje (Figura 7).

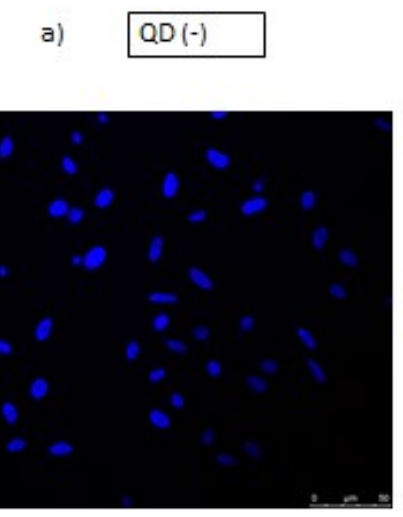
b)

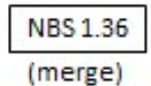

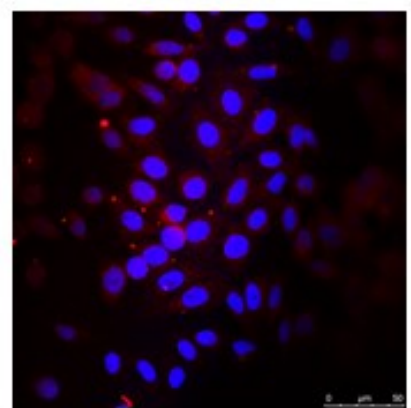

c)

NBS 1.37 (merge)

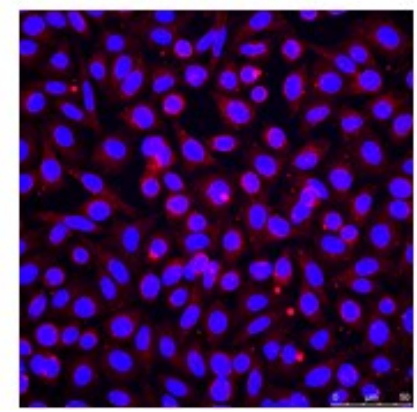

Figura 7. Inmunoensayo con QD en la línea celular HeLa. a) QD sin unión a anticuerpo como control negativo. b) Marcaje específico de GAPDH mediante el empleo del bioconjugado QD-GAPDH (NBS 1.36). c) Marcaje específico de Tubulina mediante el empleo del bioconjugado QD-Ab secundario anti-mouse (NBS 1.37).

Como otra alternativa a la falta de especificidad y penetrabilidad de los QD, se modificó el ratio QD-Ab. Originalmente se empleaban $6 \mu \mathrm{L}$ de QD y $1,5 \mu \mathrm{L}$ de anticuerpo, decidiéndose emplear por un lado 12 $\mu \mathrm{L}$ de QD con $3 \mu \mathrm{L}$ de anticuerpo y paralelamente $6 \mu \mathrm{L}$ de QD con $3 \mu \mathrm{L}$ de anticuerpo. En este caso sí se observó una notable mejoría en el marcaje tanto de GAPDH como de tubulina (Figura 8). Como resultado de este experimento se demostró la importancia de optimizar el ratio QD-Ab para obtener un marcaje por inmunofluorescencia.

a)
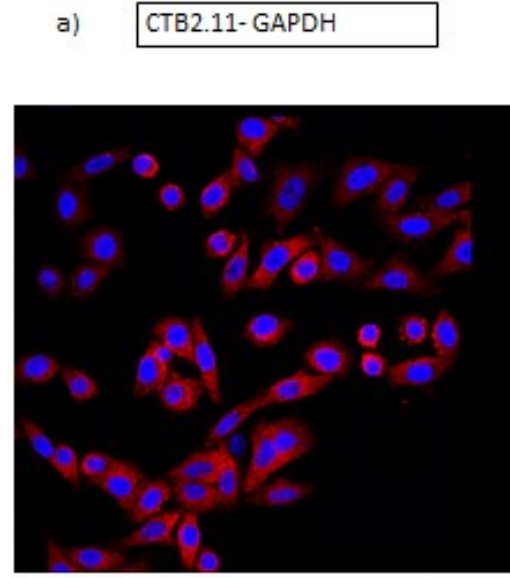

b) CTB2.11- Ab secundario

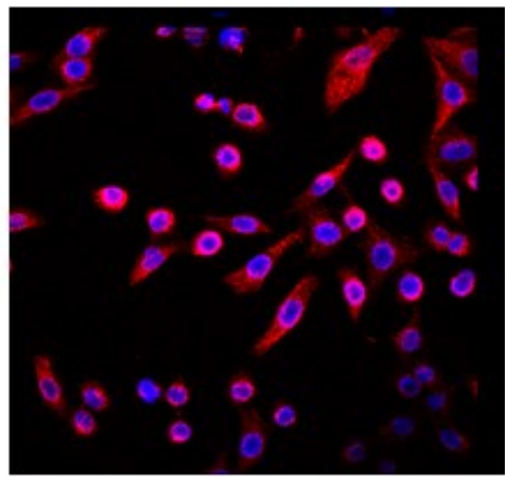

Figura 8. a) Inmunoensayo basado en QD. Marcaje de GAPDH empleando $12 \mu \mathrm{L}$ del bioconjugado CTB2.11 y $3 \mu \mathrm{ldel}$ anticuerpo. b) Inmunoensayo basado en QD. marcaje de Tubulina empleando 6 $\mu \mathrm{L}$ del bioconjugado CTB2.11 y $3 \mu 1$ del anticuerpo.

\section{Conclusiones}

Tras la realización de este trabajo, se han sintetizado sondas de QDs utilizando diferentes estrategias de conjugación para unir anticuerpos monoclonales. Así se han sintetizado sondas con diferentes proteínas adaptoras y sondas de unión covalente directa a anticuerpos. En concreto se han unido anticuerpos de histona y tubulina para comprobar la eficacia de penetrabilidad nuclear de los QDs. En este estudio se han empleado dos líneas celulares diferentes como son SH-SY5Y y HeLa en las cuales se han llevado a cabo diversos inmunoensayos para establecer que, por el momento, los QDs no están penetrando de una forma específica en el núcleo celular. Además, se ha determinado la variabilidad existente en la utilización de diferentes líneas celulares y la necesidad de optimizar para cada una de ellas el ratio de QD/Ab utilizado en la sonda.

Nuestro propósito es seguir mejorando estas técnicas para poder cuantificar tanto a nivel citosólico como nuclear, proteínas implicadas en procesos patológicos como son TDP-43 y pTDP-43 y poder establecer las bases moleculares del ELA mediante los estudios con QDs, de forma que se expandirían las aplicaciones de la inmunofluorescencia clásica. 
También se pretende poner a punto el marcaje simultáneo de varias dianas, marcando con sondas de QDs varias moléculas dentro de la misma célula para abordar así patologías como el ELA y descifrar de esta forma los complejos procesos celulares que tienen lugar en las mismas.

\section{Referencias}

1. Zrazhevskiy, P., True, L. D., \& Gao, X. 2013. Multicolor multicycle molecular profiling with quantum dots for single-cell analysis. Nature protocols, 8(10):1852.

2. Liu, E. Y., Russ, J., Cali, C. P., Phan, J. M., Amlie-Wolf, A., \& Lee, E. B. 2019. Loss of Nuclear TDP-43 Is Associated with Decondensation of LINE Retrotransposons. Cell reports, 27(5):14091421.

3. Mann, J. R., Gleixner, A. M., Mauna, J. C., Gomes, E., DeChellis-Marks, M. R., Needham, P. G., ... \& Guo, L.2019. RNA Binding Antagonizes Neurotoxic Phase Transitions of TDP-43. Neuron, 102(2):265-267.

4. Guerrero, E. N., Mitra, J., Wang, H., Rangaswamy, S., Hegde, P. M., Basu, P., ... \& Hegde, M. L. 2019. Amyotrophic lateral sclerosis-associated TDP-43 mutation Q331K prevents nuclear translocation of XRCC4-DNA ligase 4 complex and is linked to genome damage-mediated neuronal apoptosis. Human molecular genetics.1-18.

5. Zrazhevskiy, P., \& Gao, X. 2013. Quantum dot imaging platform for single-cell molecular profiling. Nature communications, 4:1619.

6. Francis, J. E., Mason, D., \& Lévy, R. 2017. Evaluation of quantum dot conjugated antibodies for immunofluorescent labelling of cellular targets. Beilstein journal of nanotechnology, 8(1):12381249.

7. Wegner, K. D., \& Hildebrandt, N.2015. Quantum dots: bright and versatile in vitro and in vivo fluorescence imaging biosensors. Chemical Society Reviews, 44(14):4792-4834.

8. East, D. A., Mulvihill, D. P., Todd, M., \& Bruce, I. J. 2011. QD-antibody conjugates via carbodiimide-mediated coupling: a detailed study of the variables involved and a possible new mechanism for the coupling reaction under basic aqueous conditions. Langmuir, 27(22):1388813896.

9. Kikkeri, R., Lepenies, B., Adibekian, A., Laurino, P., \& Seeberger, P. H. 2009. In vitro imaging and in vivo liver targeting with carbohydrate capped quantum dots. Journal of the American Chemical Society, 131(6): 2110-2112.

10. Xing, Y., Chaudry, Q., Shen, C., Kong, K. Y., Zhau, H. E., Chung, L. W., ... \& Wang, M. D. 2007. Bioconjugated quantum dots for multiplexed and quantitative immunohistochemistry. Nature protocols, 2(5):1152.

11. Aldeek, F., Hawkins, D., Palomo, V., Safi, M., Palui, G., Dawson, P. E., ... \& Mattoussi, H. 2015. UV and sunlight driven photoligation of quantum dots: understanding the photochemical transformation of the ligands. Journal of the American Chemical Society, 137(7):2704-2714.

12. Matea, C. T., Mocan, T., Tabaran, F., Pop, T., Mosteanu, O., Puia, C., ... \& Mocan, L. 2017. Quantum dots in imaging, drug delivery and sensor applications. International journal of nanomedicine, 12: 5421-5431.

13. Williams, Y., Byrne, S., Bashir, M., Davies, A., Whelan, A., Gun'Ko, Y., ... \& Volkov, Y. 2008. Comparison of three cell fixation methods for high content analysis assays utilizing quantum dots. Journal of microscopy, 232(1):91-98.

14. Bitrián, C. T., Martínez, A., \& Palomo, V. 2018.Biosensores específicos basados en quantum dots en la detección de proteínas patológicas en ELA. Dianas, 7(2):1-10. 